\title{
Asymmetry-induced effects in coupled phase-oscillator ensembles: Routes to synchronization
}

\author{
Jane H. Sheeba, ${ }^{1,2}$ V. K. Chandrasekar, ${ }^{1,2}$ Aneta Stefanovska, ${ }^{1,3}$ and Peter V. E. McClintock ${ }^{1}$ \\ ${ }^{1}$ Department of Physics, Lancaster University, Lancaster LA1 4YB, United Kingdom \\ ${ }^{2}$ Centre for Nonlinear Dynamics, School of Physics, Bharathidasan University, Tiruchirappalli, 620024 Tamilnadu, India \\ ${ }^{3}$ Faculty of Electrical Engineering, University of Ljubljana, Tržaška 25, 1000 Ljubljana, Slovenia
}

(Received 3 December 2008; published 7 April 2009)

\begin{abstract}
A system of two coupled ensembles of phase oscillators can follow different routes to interensemble synchronization. Following a short report of our preliminary results [Phys. Rev. E 78, 025201(R) (2008)], we present a more detailed study of the effects of coupling, noise, and phase asymmetries in coupled phaseoscillator ensembles. We identify five distinct synchronization regions and routes to synchronization that are characteristic of the coupling asymmetry. We show that noise asymmetry induces effects similar to that of coupling asymmetry when the latter is absent. We also find that phase asymmetry controls the probability of occurrence of particular routes to synchronization. Our results suggest that asymmetry plays a crucial role in controlling synchronization within and between oscillator ensembles, and hence that its consideration is vital for modeling real life problems.
\end{abstract}

DOI: 10.1103/PhysRevE.79.046210

PACS number(s): 05.45.Xt, 89.75.Fb, 87.19.1m

\section{INTRODUCTION}

Ensembles of coupled oscillators are ubiquitous in nature. They arise in diverse areas of science including physics, biology, chemistry, neuroscience, social, electrical, and ecological systems. Examples include synchronous emission of light pulses by populations of fireflies [1], synchronized firing of cardiac pacemaker cells [2], synchronization in ensembles of electrochemical oscillators [3,4], both short- and long-range synchronizations in the brain (within and between neuronal ensembles) [5-7], emission of chirps by a population of crickets [8], and synchronous clapping of audiences in auditoria. The dynamical properties of large ensembles of this kind have been a subject of intense interest since the 1960s [9-13]. Mean-field theory facilitates the study of such ensembles by reducing the dynamics of a number of oscillators to the dynamics of their mean field, i.e., effectively of a single oscillator. In principle, each oscillator in the ensemble contributes to the dynamics of the mean field, so that the collective dynamics of the entire ensemble can be represented by the dynamics of the mean field. This approach has a good analytical background that enables identification of bifurcation boundaries and stability criteria for understanding the synchronization dynamics of the ensemble. Although the mean-field approach suggests consideration of the dynamics of just one oscillator in place of the ensemble dynamics, recent research has identified new phenomena such as intraensemble and interensemble clusterings [14] that can only be understood in terms of ensembles. Thus one should expect to model natural systems comprised of interacting entities as ensembles of coupled oscillators rather than always approximating them as a single oscillator.

Synchronization or concurrence between oscillatory systems is a remarkable phenomenon that is often inescapable for coupled oscillators. Phase synchronization was first reported by the Dutch physicist Christiaan Huygens well back in the 17th century based on his observation of two pendulum clocks that persisted in precise antiphase, seemingly indefinitely. Thereafter, the phenomenon of synchronization has been studied theoretically $[10,11,15-17]$ and experimentally $[3-7,18-20]$ in great detail. It is well known that the control of synchronization in natural systems [21-23] is of great importance. Synchronization also occurs in, e.g., lasers and Josephson-Junction arrays [24,25], the cardiorespiratory interaction [26,27], and temporal coding and cognition via brain waves [28-32]. However the emergence of synchronized oscillations can also give rise to undesirable effects, as in the case of epileptic seizures [33,34], Parkinson tremor [35,36], or pedestrians on the Millennium Bridge [12].

In real systems, the interactions between the oscillators are often asymmetric. Examples include cardiorespiratory $[37,38]$ and cardio- $\delta$ electroencephalogram interactions [39], interactions among activator-inhibitor systems [4,40-42], coupled circadian oscillators [43], and the interactions between ensembles of oscillators in neuronal dynamics $[28,44,45]$. Neglecting coupling asymmetry, i.e., assuming symmetric interactions, is an approximation that may simplify the analysis but which may also lead to a model that fails to describe important phenomena occurring in the system. We have already reported [14] novel global clustering phenomena and novel routes to interensemble synchronization that occur only in the case of asymmetrically interacting systems. It is thus evident that explicit consideration of asymmetry in the interaction may be essential to create a realistic model.

In this paper, we supplement the preliminary account [14] of our investigations of two asymmetrically interacting ensembles of oscillators by providing additional detail of the different synchronization regimes, and we extend it by reporting the effects induced by noise asymmetry. We thereby emphasize the importance of asymmetry-in coupling, noise, and phase-in such systems. We show that it is the coupling and phase asymmetries that control their synchronization. We also report the occurrence of certain routes to interensemble synchronization. We show that these routes are characteristic of asymmetrically interacting ensembles of oscillators and that they cannot occur in systems where the interactions are symmetrical. These results yield insights into how synchronization arises in coupled oscillator ensembles. 
This understanding is an essential prerequisite for the development of control schemes, paving the way to possible methods of controlling synchronization in real systems.

We discuss the model of asymmetrically interacting ensembles of oscillators and define their mean field in Sec. II. In Sec. III we discuss analytically the stability of the incoherent (i.e., unsynchronized) state in the thermodynamic limit and consider how it can be modeled numerically. Section IV defines the five distinct synchronization regimes that we have identified and discusses in turn how each of them is influenced by asymmetry in coupling, noise, and phase. The several routes followed to synchronization, and between different synchronization regimes, are discussed in Sec. V. Finally, in Sec. VI we summarize the main results and draw conclusions.

\section{COUPLED PHASE OSCILLATOR ENSEMBLES}

The energy emitted or absorbed by an individual oscillator in the ensemble will alter the physical states of the neighbors to which it is coupled; in particular, the periods of its neighbors are altered (either lengthened or shortened). The way in which the period is altered depends on the state of the neighboring oscillator at the moment when it receives the impulse. One of the commonest scenarios to consider is an ensemble of nonlinear oscillators evolving in a globally attracting limit cycle of constant amplitude. Such oscillators are called limit cycle or phase oscillators. If they are coupled in such a way that they will not be perturbed sufficiently to leave their limit cycles, then 1 degree of freedom is enough to describe the system dynamics. Let us consider a system of two asymmetrically interacting ensembles of oscillators (AIEOs). Their phase dynamical equations can be written as [10]

$$
\begin{aligned}
\dot{\theta}_{i}^{(1,2)}= & \omega_{i}^{(1,2)}-\frac{A^{(1,2)}}{N^{(1,2)}} \sum_{j=1}^{N^{(1,2)}} f\left(\theta_{i}^{(1,2)}-\theta_{j}^{(1,2)}+\alpha^{(1,2)}\right) \\
& -\frac{B}{N^{(2,1)}} \sum_{j=1} h\left(\theta_{i}^{(1,2)}-\theta_{j}^{(2,1)}+\alpha^{(3)}\right)+\eta_{i}^{(1,2)}(t) .
\end{aligned}
$$

The interactions are characterized by coupling parameters $A^{(1,2)}$ and $B$ to quantify, respectively, the interactions within (intra) and between (inter) the ensembles; $f$ and $g$ are $2 \pi$-periodic functions that describe coupling in the ensembles. The fact that $A^{(1)} \neq A^{(2)}$ implies that the oscillators in the ensembles are asymmetrically coupled. $\theta_{i}^{(1,2)}$ are the phases of the $i$ th oscillator in each ensemble and $N^{(1,2)}$ refer to the ensemble sizes; we take $N^{(1)}=N^{(2)}=N$. From Eq. (1), it is obvious that each oscillator will run at its own characteristic frequency $\omega_{i}$ when uncoupled. However when coupled, there is a tendency for a collective behavior in the ensemble to arise. Depending on the strength of the coupling parameters, the oscillators either partially or completely synchronize. The emergence of synchronization is spontaneous beyond a critical value of the coupling parameter.

The $\eta_{i}^{(1,2)}$ are independent Gaussian white noises with $\left\langle\eta_{i}^{(1,2)}(t)\right\rangle=0$ and $\left\langle\eta_{i}^{(1,2)}(t) \eta_{j}^{(1,2)^{\prime}}(t)\right\rangle=2 K^{(1,2)} \delta\left(t-t^{\prime}\right) \delta_{i j}$ and
$K^{(1,2)}$ are the noise intensities; $K^{(1)} \neq K^{(2)}$ represents noise asymmetry. Phase asymmetry is introduced by phase shifts $0 \leq \alpha^{(1,2,3)}<\pi / 2$. The primary effect of the phase asymmetry is to synchronize the oscillators to an entrainment frequency that differs from a simple average of their natural frequencies. Such asymmetry is widespread in natural systems like heart cells [15] and the cardiorespiratory interactions [37,38]. Phase asymmetry is used to model synaptic information and time delays in neuronal networks and also in the phase reduction in nonisochronous oscillators [13]. The natural oscillator frequencies $\omega_{i}^{(1,2)}$ are assumed to be Lorentzianly distributed as $g^{(1,2)}(\omega)=\frac{\gamma}{\pi}\left[\gamma^{2}+\left(\omega^{(1,2)}-\bar{\omega}^{(1,2)}\right)^{2}\right]^{-1}$ with central frequencies $\bar{\omega}^{1,2}$ and $\gamma$ is the half width at half maximum.

\section{Mean field}

When $N \rightarrow \infty$ in the thermodynamic limit, each oscillator in the ensemble can be regarded as being coupled to the mean field. Thus for infinitely many oscillators, synchronization can conveniently be defined and characterized by a mean-field (order) parameter,

$$
r^{(1,2)} e^{i \psi^{(1,2)}}=\frac{1}{N} \sum_{j=1}^{N} e^{i \theta_{j}^{(1,2)}} .
$$

Here $\psi^{(1,2)}(t)$ are the average phases of the oscillators in the respective ensembles and $r^{(1,2)}(t)$ provide measures of the coherence of each oscillator ensemble, which varies from 0 to 1 . The amplitude of each order parameters $r^{(1,2)}$ vanishes when the oscillators in the corresponding ensemble fall out of synchronization with each other and is positive for synchronized states, thus characterizing intraensemble synchronization. When $\delta \psi=\psi^{(1)}-\psi^{(2)} \approx$ const the ensembles are mutually locked in phase, defining the state of interensemble synchronization. Geometrically, if we consider the phases of all the oscillators to be moving on the unit circle, then the mean field is the centroid of all the phases. With this characterization, we show that an increase in the coupling strength between two ensembles that are synchronized separately does not immediately result in their mutual phase locking. Rather, phase locking occurs through either one of two different routes: in route I the oscillators in the two ensembles combine and form clusters; in route II one of the ensembles desynchronizes while the other remains synchronized. Further, there also exists the possibility that phase locking between the ensembles cannot occur at all.

\section{STABILITY OF THE INCOHERENT STATE IN THE THERMODYNAMIC LIMIT}

In the limit $N \rightarrow \infty$, a density function can be defined as $\rho^{(1,2)}(\theta, t, \omega) d \omega d \theta$ to describe the number of oscillators with natural frequencies within $[\omega, \omega+d \omega]$ and with phases within $[\theta, \theta+d \theta]$ at time $t$. For fixed $\omega$ the distribution $\rho^{(1,2)}(\theta, t, \omega)$ obeys the evolution equation,

$$
\frac{\partial \rho^{(1,2)}}{\partial t}=-\frac{\partial}{\partial \theta}\left(\rho^{(1,2)} v^{(1,2)}\right)+K^{(1,2)} \frac{\partial^{2} \rho^{(1,2)}}{\partial \theta^{2}},
$$

where $v^{(1,2)}$ are given by 


$$
\begin{aligned}
v^{(1,2)}= & \omega^{(1,2)}-A^{(1,2)} \int_{0}^{2 \pi} d \theta \int_{-\infty}^{\infty} g^{(1,2)}(\omega) \\
& \times f\left(\theta-\phi+\alpha^{(1,2)}\right) \rho^{(1,2)}(\phi, t, \omega) d \omega-B \int_{0}^{2 \pi} d \theta \\
& \times \int_{-\infty}^{\infty} g^{(2,1)}(\omega) h\left(\theta-\phi+\alpha^{(3)}\right) \rho^{(2,1)}(\phi, t, \omega) d \omega .
\end{aligned}
$$

The function $\rho^{(1,2)}(\theta, t, \omega)$ is real and $2 \pi$ periodic in $\theta$, so it can be expressed as a Fourier series in $\theta$,

$$
\begin{aligned}
\rho^{(1,2)}(\theta, t, \omega) & =\sum_{l=-\infty}^{\infty} \rho_{l}^{(1,2)}(\omega, t) e^{i l \theta} \\
& =\frac{1}{2 \pi}+\rho_{1}^{(1,2)} e^{i \theta}+\text { c.c. }+\eta(\theta, t, \omega),
\end{aligned}
$$

where c.c. is the complex conjugate of the preceding term and $\eta(\theta, t, \omega)$ denotes the second and higher harmonics. Substituting $\rho^{(1,2)}(\theta, t, \omega)$ into the evolution equation, we get

$$
\dot{\rho}_{l}^{(1,2)}+\left(i l \hat{\omega}^{(1,2)}+l^{2} K^{(1,2)}\right) \rho_{l}^{(1,2)}=2 i l \pi \sum_{k=1}^{\infty}\left(a_{k} \rho_{l-k}^{(1,2)}+a_{k}^{*} \rho_{l+k}^{(1,2)}\right),
$$

where $\rho_{-l}^{(1,2)}=\rho_{l}^{*(1,2)}, \quad \hat{\omega}^{(1,2)}=\omega^{(1,2)}-\left(A^{(1,2)} f_{0}+B h_{0}\right), \quad$ and $a_{k}$ $=\left(A^{(1,2)} e^{i k \alpha^{(1,2)}} f_{k}\left\langle\rho_{k}^{(1,2)}\right\rangle+B e^{i \alpha^{(3)}} h_{k}\left\langle\rho_{k}^{(2,1)}\right\rangle\right)$. The linearized form of Eq. (2) reads as

$$
\dot{\rho}_{k}^{(1,2)}=-\left(i k \hat{\omega}^{(1,2)}+k^{2} K^{(1,2)}\right) \rho_{k}^{(1,2)}+i k a_{k},
$$

where the Fourier components for $|l|>k$ are neglected since $l= \pm k$ are the only nontrivial unstable modes, $\rho_{0}=1 / 2 \pi$ is the trivial solution corresponding to incoherence, and $f_{k}$ and $h_{k}$ are coefficients of the Fourier series of functions $f$ and $h$. Here $\langle\cdot\rangle$ represents the average over the frequencies $\omega^{(1,2)}$ weighted by the Lorentzian distribution $g^{(1,2)}(\omega)$. Solving Eq. (3) we get

$$
\rho_{k}^{(1,2)}=b_{k}^{(1,2)}(\omega) e^{\lambda_{k} t}+O(|\rho|) .
$$

Substituting the above equation back into Eq. (3) we find

$$
b_{k}^{(1,2)}(\omega)=\frac{\left(\bar{A}^{(1,2)}\left\langle b_{k}^{(1,2)}\right\rangle+\bar{B}\left\langle b_{k}^{(2,1)}\right\rangle\right)}{\left(\lambda_{k}+i k \hat{\omega}^{(1,2)}+k^{2} K^{(1,2)}\right)},
$$

where $\bar{A}^{(1,2)}=i k A^{(1,2)} f_{k} e^{i k \alpha^{(1,2)}}$ and $\bar{B}=i k B h_{k} e^{i k \alpha^{(3)}}$. The integrals in this equation can be written as constants $C^{(1,2)}$ which are to be determined in a self-consistent manner. Thus, for the assumption

$$
C_{k}^{(1,2)}=\int_{-\infty}^{\infty} b_{k}^{(1,2)}\left(\omega^{\prime}\right) g^{(1,2)}\left(\omega^{\prime}\right) d \omega^{\prime},
$$

equation (5) for $b_{k}^{(1,2)}$ becomes

$$
b_{k}^{(1,2)}(\omega)=\frac{\left(A^{(1,2)} C_{k}^{(1,2)}+B C_{k}^{(2,1)}\right)}{\left(\lambda_{k}+i k \hat{\omega}^{(1,2)}+k^{2} K^{(1,2)}\right)} .
$$

This on substitution back into Eq. (5) results in the following characteristic equation:

$$
1=\bar{A}^{(1)} m_{1}+\bar{A}^{(2)} m_{2}-\left(\bar{A}^{(1)} \bar{A}^{(2)}-\bar{B}^{2}\right) m_{1} m_{2},
$$

where $m_{i}=\int_{-\infty}^{\infty}\left[g^{(i)}(\omega) d \omega\right] /\left(\lambda_{k}+i k \hat{\omega}^{(i)}+k^{2} K^{(i)}\right), \quad i=1,2$. The eigenvalues obtained from Eq. (8) are

$$
\begin{aligned}
\lambda_{k \pm}= & \frac{\bar{A}^{(1)}+\bar{A}^{(2)}}{2}-\gamma-k^{2} \bar{K}-i k \bar{\omega} \pm \frac{1}{2}\left[4 \bar{B}^{2}+\Delta A^{2}-k(\Delta \omega\right. \\
& -i k \Delta K)(\Delta \omega-i k \Delta K+2 i \Delta A)]^{1 / 2}
\end{aligned}
$$

where $\bar{K}=\frac{\left(K^{(1)}+K^{(2)}\right)}{2}, \quad \Delta K=K^{(1)}-K^{(2)}, \quad \Delta \omega=\bar{\omega}^{(1)}-\bar{\omega}^{(2)}, \quad \Delta A$ $=\left(\bar{A}^{(1)}-\bar{A}^{(2)}\right)$, and $\bar{\omega}=\left(\bar{\omega}^{(1)}+\bar{\omega}^{(2)}\right) / 2$.

For a detailed analysis of the above equation, we specify sinusoidal forms for the functions $f$ and $h$ as $\{f, h\}(\theta)$ $=\sin \theta$. Therefore the eigenvalue [Eq. (9)] becomes

$$
\begin{aligned}
\lambda_{ \pm}= & -\gamma-\bar{K}+\frac{\kappa}{4} e^{i \alpha} \pm \frac{1}{2}\left[\xi e^{2 i \alpha}-\hat{A}(\Delta K+i \Delta \omega) e^{i \alpha}-\Delta \omega^{2}\right. \\
& \left.+\Delta K^{2}+2 i \Delta \omega \Delta K\right]^{1 / 2}-i \bar{\omega}
\end{aligned}
$$

or equivalently we have

$\lambda_{ \pm}$

$$
= \begin{cases}-\bar{K}-\gamma+\frac{\kappa}{4} e^{i \alpha} \pm \frac{1}{2}\left(p^{2}+q^{2}\right)^{1 / 4} \exp \left(i \frac{1}{2} \zeta\right)-i \bar{\omega}, & p>0 \\ -\bar{K}-\gamma+\frac{\kappa}{4} e^{i \alpha} \pm \frac{i}{2}\left(p^{2}+q^{2}\right)^{1 / 4} \exp \left(i \frac{1}{2} \zeta\right)-i \bar{\omega}, & p<0\end{cases}
$$

where $\alpha_{i}=\alpha, \quad i=1,2,3, \quad \kappa=A^{(1)}+A^{(2)}, \quad \hat{A}=\left(A^{(1)}-A^{(2)}\right), \quad \xi$ $=\left(\frac{1}{4} \hat{A}^{2}+B^{2}\right), \quad \zeta=\tan ^{-1}\left(\frac{q}{p}\right), \quad p=\xi \cos (2 \alpha)+\hat{A}[\Delta \omega \sin \alpha$ $-\Delta K \cos \alpha]-\Delta \omega^{2}+\Delta K^{2}, \quad$ and $\quad q=\xi \sin (2 \alpha)-\hat{A} \Delta \omega \cos \alpha$ $-\hat{A} \Delta K \sin \alpha+2 \Delta \omega \Delta K$. The resultant bifurcation diagram is shown in Fig. 1. It is discussed in detail below, in Secs. IV A and IV C. Here it is obvious that for the case when phase asymmetry is absent, when $A^{(2)}=B=0$, the characteristic [Eq. (8)] reduces to the characteristic equation of the Kuramoto model derived by Kuramoto [10] and Strogatz [11].

\section{A. Numerical considerations}

To investigate the system numerically, we use a RungeKutta fourth-order (RK4) routine for solving the model equations with a time step of 0.01 ; we have confirmed that the results are unaffected by decreasing the time step below 0.01 . We take $N=1000$ in each ensemble and the initial phases of the oscillators are assumed to be equally distributed within the interval $[0,2 \pi]$. As a signature of synchronization, we take the condition $\operatorname{Re}\left(\lambda_{ \pm}\right)>0$ in the case of the analytic treatment. For the numerical experiment, we set $r^{(1,2)}>0.7$ for intraensemble synchronization in the corresponding ensembles and a constant $\delta \psi$ for interensemble synchronization as the conditions. The numerical condition for intraensemble synchronization, that $r^{(1,2)}>0.7$, may at first seem too strict when compared with the analytic condition that $r^{(1,2)}>0$. However, there are certain differences between analytic and numeric considerations that make this choice reasonable. Mainly, $N$ is finite for the numerical experiment, whereas analytic conditions are derived in the limit $N \rightarrow \infty$. Further, the analytic and numeric bifurcation bound- 

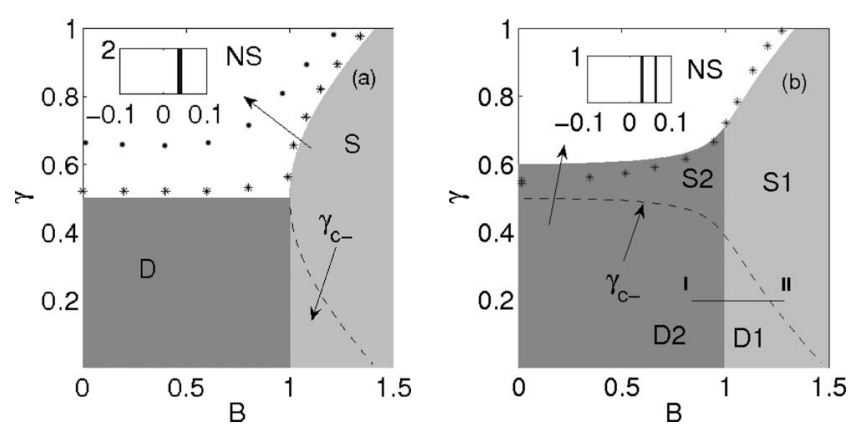

FIG. 1. Theoretical $B-\gamma$ bifurcation diagram for $\alpha=0, \Delta \omega=1$, and $K^{(1,2)}=0$, with (a) $A^{(1)}=A^{(2)}=1$ and (b) $A^{(1)}=1.2, A^{(2)}=1.0$. The different synchronization regimes are as follows: NS, no synchronization; S, synchronization with single entrainment frequency (reached via a single Hopf bifurcation); D, synchronization with two entrainment frequencies (two Hopf bifurcations); S1, both the ensembles entrained to a single frequency; S2, either of one of the ensembles synchronized with single entrainment frequency; D1, the two ensembles behave as one, with the oscillators in each ensemble entrained to either of the two distinct frequencies; D2, synchronization in both the ensembles separately with two entrainment frequencies. Regardless of symmetry, the notations S and D represent single or double frequencies, respectively, occurring after one or two Hopf bifurcations. The boundary between regimes NS and S/D represents $\gamma_{c+}$. The lines of $*$ s represent the numerically determined bifurcation boundaries for $r>0.7$. For comparison, a numerical boundary $(\bullet \mathrm{s})$ for $r>0.5$ is also plotted in (a). Insets show the frequency distributions (also obtained numerically) for the indicated regions; their ordinate axes represent oscillator counts in thousands. Note that the occurrence of perfect synchronization with (a) 2000 and (b) 1000 oscillator groups cannot be expected throughout the whole of each indicated region. Line I-II in (b) is one of the routes to synchronization discussed in Sec. V.

aries (discussed later) are found to match quite closely for this choice of the numeric threshold for $r^{(1,2)}$. We have plotted the numerical boundary for $r=0.5$ along with $r=0.7$ in Fig. 1(a) to illustrate this.

\section{SYNCHRONIZATION REGIMES}

We have identified analytically the possibility of five distinct dynamical regimes [14]:

(i) NS: the region of no synchronization or incoherence (steady state).

(ii) S1: the region of global (interensemble) synchronization, in which the oscillators of both ensembles are all entrained to the same frequency.

(iii) S2: the region where there is synchronization within one ensemble but not the other.

(iv) D2: the region of synchronization within both ensembles, separately and independently, with two different entrainment frequencies.

(v) D1: a global regime in which the two ensembles behave as one, but the oscillators within each ensemble are entrained at either one of two distinct entrainment frequencies. We will call this phenomenon interensemble clustering.

Regions S2 and D1 cannot occur when coupling and noise asymmetries are absent $[46,47]$ [see Fig. 1(a)]. In Secs.
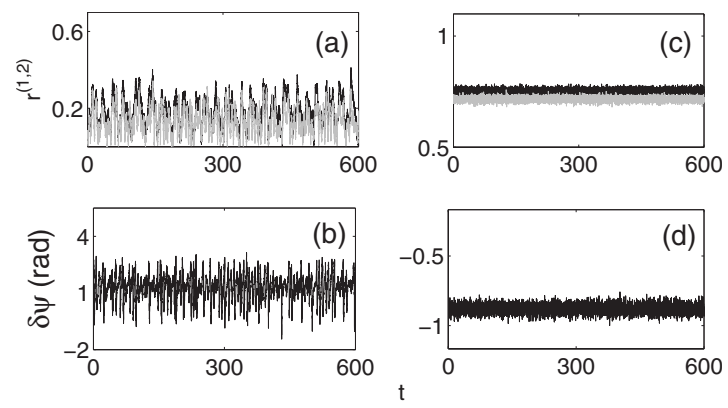

FIG. 2. Time variations in the coherence parameters $r^{(1)}$ (gray), $r^{(2)}$ (black), and the phase difference $\delta \psi$, as obtained from numerical simulations. Parameter values are [(a) and (b)] $B=1, \alpha=0.23$ and [(c) and (d)] $B=1, \alpha=0.47$ corresponding to regions D1 and D2, respectively, of Fig. 1(b) when one travels along line I-II. Note that in region D1 the order parameters display no synchronization.

IV A-IV C, we will consider how these synchronization regimes are affected by coupling, noise, and phase asymmetries, respectively.

\section{A. Effect of coupling asymmetry}

Consider Fig. 1(b) for the case $\alpha=0$ and $K^{(1)}=K^{(2)}=0$ when $\xi-\Delta \omega^{2}>0$. If we start from the state of no synchronization (region NS) and decrease $\gamma$ for fixed $B>1$, the incoherent (steady) state becomes unstable via a single Hopf bifurcation. Thus the system enters into the region $\mathrm{S} 1$ from NS (crossing $\gamma_{c+}$ ) and the ensembles entrain to a single frequency $\Omega_{+}$. With further decrease in $\gamma$ below the $\gamma_{c-}$ line in the region D1 (in Fig. 1), a new entrainment frequency emerges through a second Hopf bifurcation. In this region, the oscillators from the two ensembles combine and form two clusters (interensemble clustering) oscillating with two frequencies,

$$
\begin{aligned}
\Omega_{ \pm}= & -\operatorname{Im}\left(\lambda_{ \pm}\right) \\
= & \pm(1 / 2)\left[\left(\xi-\Delta \omega^{2}\right)^{2}+\hat{A}^{2} \Delta \omega^{2}\right]^{1 / 4} \\
& \times \sin \left(\frac{1}{2} \tan ^{-1}\left[\hat{A} \Delta \omega /\left(\xi-\Delta \omega^{2}\right)\right]\right)+\bar{\omega} .
\end{aligned}
$$

The lines $\gamma_{c_{ \pm}}$in Fig. 1 are obtained by imposing the condition $\operatorname{Re}\left(\lambda_{ \pm}\right)=0$ in Eq. (11).

Thus in this region the order parameters $r^{(1,2)}$ either fluctuate in a quasiperiodic manner or have complicated dynamics [see Figs. 2(a) and 2(c)]. This is because each ensemble has two clusters oscillating with different frequencies [see Figs. 2(a) and 3]. Thus, the behavior of the order parameters $r^{(1,2)}$ is quite subtle. Since $r^{(1,2)}$ measure only the amount of synchronization within an ensemble, a decrease in $r^{(1,2)}$ will not necessarily mean desynchronization. Rather, for a sufficient value of the coupling parameters, a decrease in $r^{(1,2)}$ represents a signature of interensemble synchronization: clustering corresponds to the occurrence of desynchronization within an ensemble because some of its oscillators tend to synchronize with the other ensemble.

Again looking at Fig. 1(b), when $B<1$, a decrease in $\gamma$ takes the system from region NS to region $\mathrm{S} 2$ by crossing the 


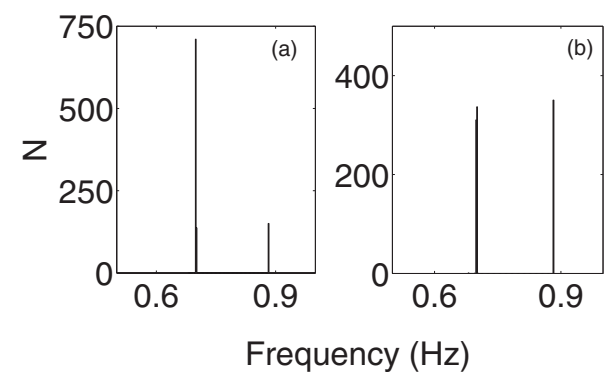

FIG. 3. The distribution of frequencies in region D1 for the same parameter values as in Fig. 2(a): (a) first ensemble; (b) second ensemble. The splitting of the first frequency component into two almost indistinguishable subcomponents corresponds to a discrepancy between numerics and analytics, attributable to approximations (see text) made in the former.

line $\gamma_{c+}$ through a single Hopf bifurcation. Further decrease in $\gamma$ causes the system to cross the line $\gamma_{c-}$ and, via another Hopf bifurcation, enter into region D2 where there are two entrainment frequencies $\Omega_{ \pm}$. The latter can be calculated from Eq. (11). In region $\mathrm{S} 2$, intraensemble synchronization can occur in either one of the ensembles, depending on whether $A^{(1)}$ or $A^{(2)}$ is greater; in Fig. 1(b), since $A^{(1)}>A^{(2)}$, synchronization occurs in the first ensemble with the second ensemble remaining incoherent. Note that, on increasing $B$ (for fixed $\gamma$ ) while in region $\mathrm{S} 2$, the condition $\xi-\Delta \omega^{2}<0$ is violated and the ensembles enter into the phase-locked region $\mathrm{S} 1$. In region $\mathrm{D} 2$, the ensembles synchronize separately to two locking frequencies [unlike region D1 where the ensembles combine and synchronize to either one of the two locking frequencies given by Eq. (12)].

The corresponding $(B-\gamma)$ bifurcation diagram for the case $A^{(1)}=A^{(2)}$ is plotted in Fig. 1(a) to show the difference between these two cases. Region D represents intraensemble synchronization which occurs through a degenerate Hopf bifurcation (similar to the route to D2) with entrainment frequencies $\Omega_{ \pm}=\mp(1 / 2)\left(\Delta \omega^{2}-B^{2}\right)^{1 / 2}+\bar{\omega}$ and $S$ represents interensemble synchronization through a single Hopf bifurcation (similar to the route to S1) with same frequency $\Omega=\bar{\omega}$. Note that regions S2 and D1 cannot arise for the symmetric coupling case and that these two synchronization regimes are therefore induced by coupling asymmetry(see Fig. 4).

The presence of two entrainment frequencies in region D1 can be seen by looking at the frequencies into which all the individual oscillators are grouped as shown in Fig. 3 (since the order parameters do not reveal this synchronization phenomenon). The interensemble clustering that occurs in this case is quite different from the formation of clusters in a single ensemble $[10,48,49]$ - here the oscillators in two different ensembles combine and form clusters. The occurrence of this phenomenon provides an insight into possible ways of controlling synchronization in more realistic situations (considering asymmetry) like neural networks where some neurons from one ensemble (say cortex) tend to synchronize with other ensemble (say thalamus) creating desirable (temporal coding) or undesirable effects (as in the case of epileptic seizures). For instance, in a thalamocortical model of the neuronal synchronization mechanisms during anesthesia

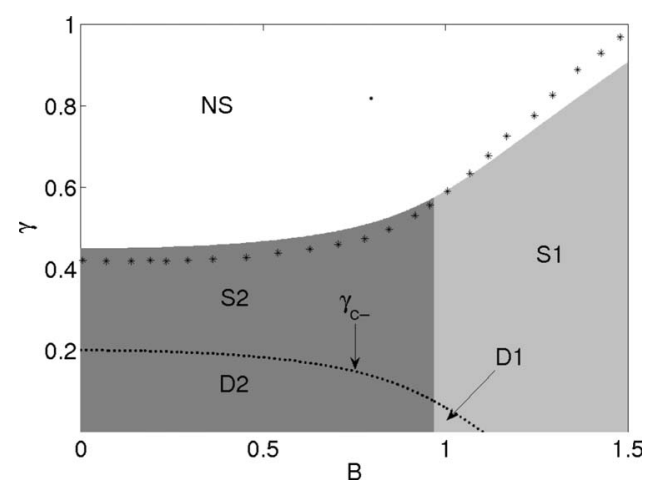

FIG. 4. Theoretical $B-\gamma$ bifurcation diagram for asymmetric noise with $A^{(1)}=A^{(2)}=1, \Delta K=0.25$ with $K^{(1)}=0.3$ and $K^{(2)}=0.05$, $\Delta \omega=1$. Note that the synchronization regimes S2 and D1 emerge in the presence of noise asymmetry even for symmetric coupling [cf. Fig. 1(a)].

[32], we found that the transition from the deep to the light anesthetized state occurs as the result of a fraction of the thalamic neurons entering into synchronization with the cortex; at the same time, of course, these thalamic neurons lose synchronization with the other thalamic neurons. The clustering that occurs in this case is desirable in the sense that it favors coding of sensory information and helps the brain to resist the effects of anesthesia by maintaining consciousness and cognition. Without coupling asymmetry, these phenomena would not occur.

\section{B. Effect of noise asymmetry}

It is well known that real physical systems are in general subject to noise. Here, we regard as "noise" any kind of random fluctuation in the system, whether originating internally or externally. Synchronization effects induced or modified by noise are of particular interest [50-54].

When asymmetric noise in introduced into a system with asymmetric coupling, the bifurcation regimes remain the same in the presence of coupling and phase asymmetries. There may be changes in the boundaries of the respective regions and their entrainment frequencies. However, for the case of symmetric coupling, asymmetric noise can induce the phenomenon of global clustering. We have already seen in Sec. IV A that interensemble clustering phenomena (region D1) cannot occur in a system with symmetric coupling and symmetric noise. Figure 5 plots the individual oscillator phases, determined numerically, indicating the transition from $\mathrm{S} 1$ to $\mathrm{D} 1$ induced by noise asymmetry. When $A^{(1)}$ $=A^{(2)}=1.4, B=1, \gamma=0.05$, and $\Delta K=0$ the system is in region $\mathrm{S}$ [corresponding to Fig. 1(a)] where synchronization occurs in both the ensembles with one entrainment frequency. This can be seen from the top panel of Fig. 5 where oscillators from both ensembles lock to form a single major cluster. On the other hand, when $\Delta K=0.25$, the combined system of the two ensembles synchronizes to two main clusters, each of which comprises a fraction of the oscillators from both ensembles [see Fig. 5 (bottom)], representing region D1. Thus asymmetric noise in some ways imitates the effects of asymmetric coupling in the absence of noise. In general, however, 


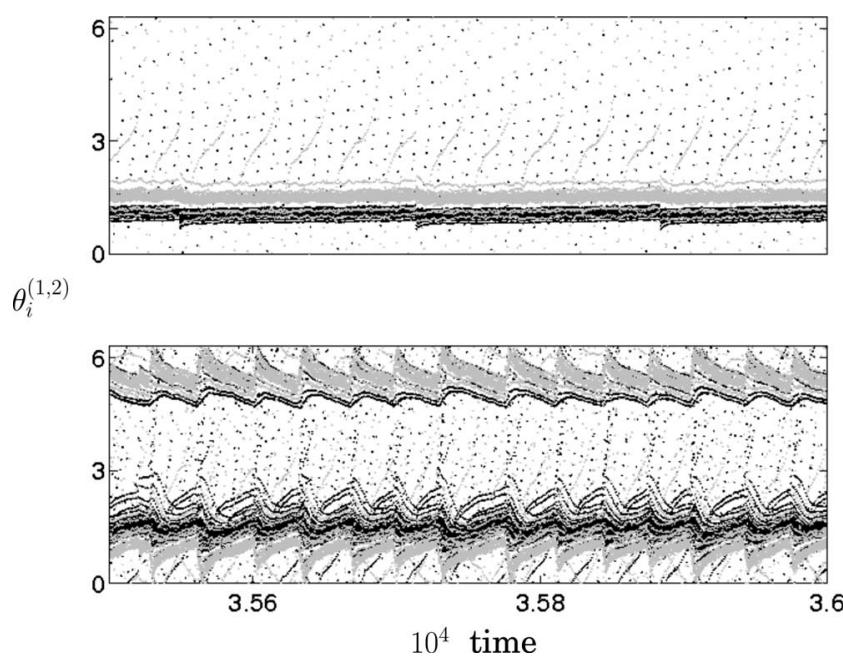

FIG. 5. Time evolution of the oscillator phases in the first (gray) and second (black) ensembles. Parameter values are $A_{1}=A_{2}=1.4$, $B=1, \gamma=0.05$, and either $\Delta K=0$, with $K^{(1)}=0.2$ and $K^{(2)}=0.2$ (top), or $\Delta K=0.25$, with $K^{(1)}=0.45$ and $K^{(2)}=0.2$ (bottom). Thus the top and the bottom panels represent the synchronization regions $S$ and D1, respectively, induced by noise asymmetry.

the coupling strength and noise intensity compete with each other in the sense that gradually increasing the coupling strength from zero will eventually favor synchronization, whereas increasing the noise intensity will favor desynchronization.

The $\mathrm{S} 2$ region also appears in this case, induced by noise asymmetry. Here too, depending on whether $\Delta K$ is positive or negative, synchronization occurs either in the second or the first ensemble, respectively, similar to the case when region $\mathrm{S} 2$ arises in the presence of coupling asymmetry. Thus noise asymmetry plays a similar role to coupling asymmetry for the symmetric coupling case, and the $(B-\gamma)$ bifurcation diagrams [Figs. 1(b) and 4] look similar. Figure 6 depicts the
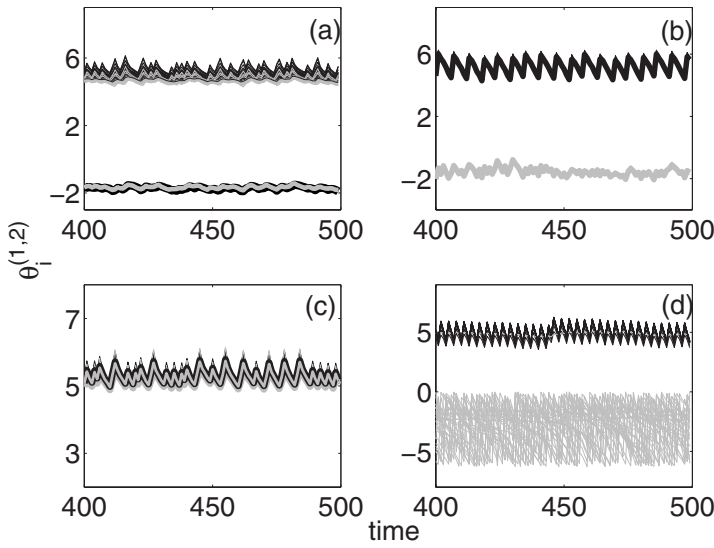

FIG. 6. Noise asymmetry-induced synchronization regimes obtained numerically for the case of symmetric coupling. Gray and black lines represent the time evolution of the oscillator phases in the first and second ensembles, respectively, for the same parameter values as in Fig. 4 and (a) $B=1, \gamma=0.1$; (b) $B=0.5, \gamma=0.1$; (c) $B$ $=1.2, \gamma=0.4$; and (d) $B=0.6, \gamma=0.4$. Panels (a) - (d) represent the synchronization regimes D1, D2, S1, and S2, respectively. results of numerical investigation of all the synchronization regimes in the presence of noise asymmetry corresponding to the analytical bifurcation diagram in Fig. 4. In contrast, for symmetric noise the dynamics is unaffected, no matter whether coupling and phase asymmetries are present or absent. The only difference is that the incoherent state becomes unstable for larger values of the critical parameters as one increases noise intensity.

\section{Effect of phase asymmetry}

For the case $\alpha \neq 0$, the interensemble regions D1 and S1 shrink as $\alpha$ increases, whereas the intraensemble synchronization region $\mathrm{S} 2$ expands, as shown in Fig. 7. This means that finite phase asymmetry reduces the probability of interensemble synchronization (note reduced S1 and D1 regions in Fig. 7) and mostly allows only intraensemble synchronization of one or both of the ensembles. For a given set of parameters, on increasing $\alpha$ from 0 , the following condition is satisfied:

$$
\xi \cos (2 \alpha)+\hat{A} \Delta \omega \sin \alpha-\Delta \omega^{2}>0
$$

up to a critical value of $\alpha=\alpha_{j}$ given by

$$
\alpha_{j}=\sin ^{-1}\left[\frac{\hat{A} \Delta \omega \pm\left[8 \xi^{2}-\Delta \omega^{2}\left(\hat{A}^{2}+B^{2}\right)\right]^{1 / 2}}{4 \xi}\right],
$$

where again, for a given set of parameters, there can only be one value of $\alpha$ that satisfies $0<\alpha \leq \pi / 2$. Upon crossing $\alpha_{j}$, condition (13) is violated and the following condition is satisfied: $\xi \cos (2 \alpha)+\hat{A} \Delta \omega \sin \alpha-\Delta \omega^{2}<0$.

As a result, when $\alpha>\alpha_{j}$ the interensemble synchronization breaks down and the system enters into a state of intraensemble synchronization. Thus as one travels from S1 (D1) to S2 (D2) across $\alpha_{j}$ the combined synchronization with single (double) frequency breaks between the ensembles and independent synchronization with single (double) frequency regime appears. Region $\mathrm{S} 2$, unlike region $\mathrm{S}$ in Fig. 1(a), embraces two states, (i) synchronization in ensemble 1 with ensemble 2 incoherent and (ii) synchronization in ensemble 2 with ensemble 1 incoherent, but does not distinguish between them.

Further, there is a critical value of $\alpha=\alpha$ above which the collective oscillations disappear and the incoherent state becomes stabilized (see Figs. 7 and 8 ). Thus by reducing the chances of occurrence of interensemble synchronization and favoring intraensemble synchronization, phase asymmetry plays a crucial role in determining the route to synchronization. Thus, for instance, in a particular problem, if one wants to have (avoid) the phenomenon of interensemble clustering (region D1) then it is obvious that phase asymmetry should be absent (finite and large).

The discrepancy between the numerically and analytically obtained boundaries in Fig. 7 is attributable to the influence of phase asymmetry. This affects region S2 which is large here [cf. Fig. 1(b) where both S2 and the discrepancy are smaller] and it changes the thresholds for $r^{(1)}$ and $r^{(2)}$. Note that neither numerics nor analytics provides an exact result. The analytic boundary is obtained from the condition $r^{(1,2)}$ 

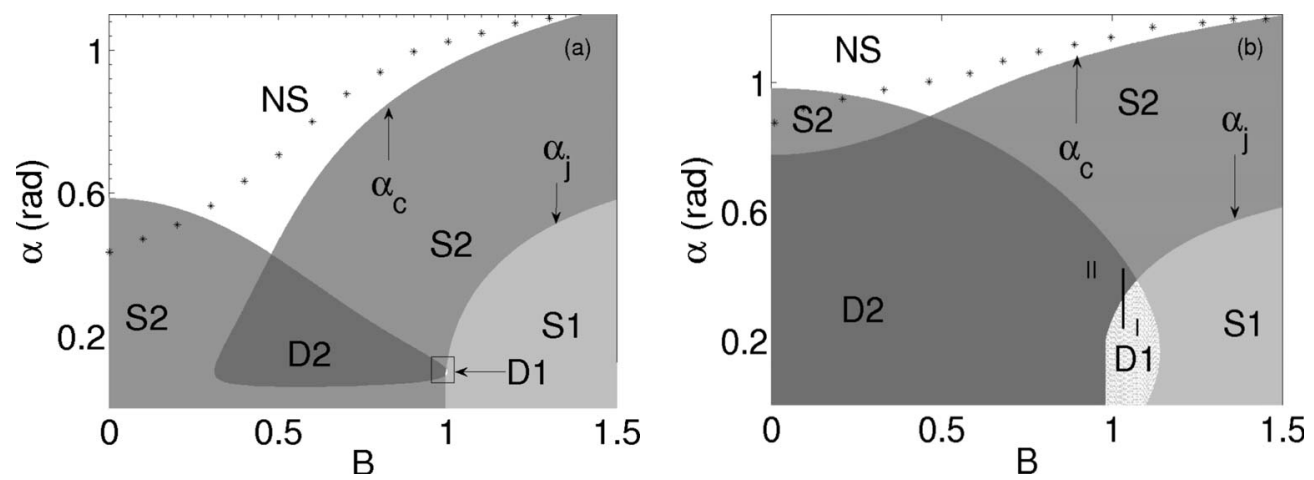

FIG. 7. Theoretical $B$ - $\alpha$ bifurcation diagram for (a) $A^{(1)}=1.2, A^{(2)}=1$, (b) $A^{(1)}=1.8, A^{(2)}=1.4$ and $\Delta \omega=1, \gamma=0.5$. The line of $*$ s represents the numerically obtained bifurcation boundary between the synchronized and incoherent states. Greatly reduced S1 and D1 regions occur due to the presence of phase asymmetry. The discrepancy between numerical and analytic boundaries is discussed in the text.

$>0$ and refers to the limit of infinitely many oscillators. For numerics, the (asterisked) boundary is obtained from the condition $r^{(1,2)}>0.7$ and refers to a finite number of oscillators.

\section{Stability of the fully synchronized states in the limit $\gamma=0$}

In this section we focus on the noise-free case with a frequency distribution that has an infinitely sharp peak. In this case, the dynamics is reduced to that of two ensembles of identical oscillators (the oscillators within the ensembles are identical while the ensembles themselves are nonidentical). Now the $\delta \psi$ corresponding to intraensemble synchronization can be obtained from Eq. (1) as

$$
\delta \psi=\sin ^{-1}[(\Delta \omega-\hat{A} \sin \alpha) / 2 B \cos \alpha] .
$$

A linear stability analysis of Eq. (1) then gives $N-1$ degenerate eigenvalues for each ensemble, namely,

$$
\lambda_{ \pm}=-A^{(1,2)} \cos \alpha-B \cos ( \pm \delta \psi+\alpha)<0,
$$

which characterize the stability of the intraensemble synchronized states of ensembles 1 and 2 . In addition, two ei-
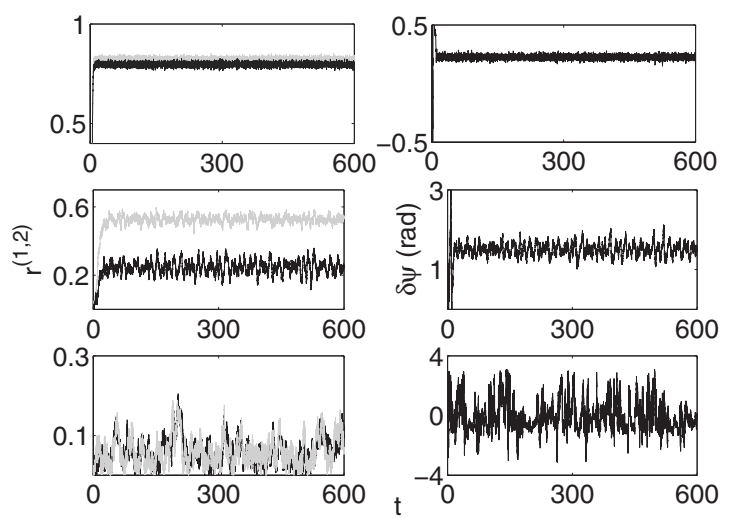

FIG. 8. The coherence parameters $r^{(1)}$ (gray) and $r^{(2)}$ (black) and the phase difference $\delta \psi$, plotted as functions of time, obtained from numerical simulations. Parameter values were: top panel, $B=0.7$, $\alpha=0.2$; middle panel, $B=0.7, \alpha=\pi / 4$ (near $\alpha_{c}$ ); and bottom panel, $B=0.7, \alpha=1.2,\left(\alpha>\alpha_{c}\right)$, corresponding to regions D2 near the NS/S2 boundary and NS, respectively [see Fig. 7(a)]. genvalues $\lambda_{0}=0$ and $\lambda_{c}=-2 B \cos \alpha \cos \delta \psi$ characterize the stability of interensemble synchronization. Hence the transition between interensemble and intraensemble synchronization states occurs at the following bifurcation point:

$$
B_{c}=(\Delta \omega-\hat{A} \sin \alpha) / 2 \cos \alpha .
$$

Note that $\delta \psi$ varies from $-\pi / 2$ to $\pi / 2$ as $\alpha$ increases. For $\alpha<\alpha_{h}$ (not shown in figures), the stability condition is satisfied by both ensembles and so intraensemble synchronization occurs in both ensembles. When $\alpha \geq \alpha_{h}$ the stability condition is violated by either one of the ensembles and at that point a Hopf bifurcation occurs. As a consequence, intraensemble synchronization occurs in one of the ensembles. For the case $A^{(1)}=A^{(2)}$, when $\delta \psi$ varies from $\pi / 2$ to 0 for increasing $\alpha$ and when $\alpha \geq \alpha_{h}$ the stability condition is violated by the first ensemble. On the other hand, when $\delta \psi$ varies from 0 to $-\pi / 2$ with increasing $\alpha$, the stability condition is violated by the second ensemble above $\alpha_{h}$.

\section{ROUTES TO SYNCHRONIZATION}

Given that the system possesses distinct synchronization regimes, it is of interest to investigate the routes it follows to synchronization. As one would expect, the route depends on the coupling, noise, and phase asymmetries. In particular, in the presence of coupling asymmetry, we have identified the following routes [14], grouped into the two different cases $\alpha=0$ and $\alpha \neq 0$, and assuming that we increase the interensemble coupling parameter $B$ keeping all the other parameters fixed. When $\alpha=0$ we find that there are at least three typical routes:

(1) The oscillators in the ensembles pass from the synchronization regime D2 through D1 to the region S1. Thus when the ensembles are synchronized separately, increasing $B$ results in interensemble clustering which then leads to interensemble synchronization or phase locking between the ensembles. This route is represented by line I-II of Fig. 1(b).

(2) There is also a possibility that when the ensembles are synchronized separately and when $B$ is increased, the intraensemble synchronization be destroyed in one of the ensembles, which on further increase in $B$ leads to phase locking between the ensembles. Thus when the system is in 
region D2 increasing $B$ causes the system to pass through the region $\mathrm{S} 2$ to region $\mathrm{S} 1$. Interensemble clustering does not occur in this route.

(3) If the ensembles are initially not synchronized (that is in region NS), then increasing $B$ can cause phase locking of the ensembles directly. Thus the system can pass directly from region NS to $\mathrm{S} 1$. This route is characteristic of the case $\alpha=0$ and cannot occur in the presence of phase asymmetry.

In the presence of phase asymmetry, i.e., $\alpha \neq 0$, the ensembles can follow any of the following routes to synchronization:

(1) The ensembles pass from region D2 through D1 to S1. This route is similar to route 1 that occurs for the case $\alpha$ $=0$. Note that when $A^{(1)}=A^{(2)}$ or $\Delta \omega=0$ only one entrainment frequency exists below $\gamma_{c-}$ and therefore this route does not occur for either case (due to the nonoccurrence of region D1).

(2) The ensembles pass from region D2 through S2 to S1. This route does not incorporate the state of interensemble clustering.

(3) When the ensembles are synchronized separately (in region D2), increasing $B$ causes the disruption of synchronization in one of the ensembles leading to synchronization in the other ensemble (region S2). Thus the ensembles pass from region $\mathrm{D} 2$ to $\mathrm{S} 2$. This route is characteristic of phase asymmetry and cannot occur for the case $\alpha=0$.

(4) If the ensembles are not synchronized, increasing $B$ will result in synchronization in either one of the ensembles. Thus the ensembles pass from regions NS to S2 (unlike NS to $\mathrm{S} 1$ in the absence of phase asymmetry).

Knowledge of these routes to synchronization is obviously important for the control of synchronization in real systems.

\section{DISCUSSION AND CONCLUSIONS}

One might intuitively suggest that the synchronization phenomena induced by coupling and noise asymmetries could also be obtained by choosing a sufficiently large difference between the mean frequencies of the two ensembles. However, the synchronization phenomena corresponding to the D1 and S2 regions can only be explained by introducing either coupling or noise asymmetries. As an illustration let us consider the eigenvalue for the noise-free case without coupling and phase asymmetries for $\Delta \omega^{2}>B^{2}$,

$$
\lambda_{ \pm}=-\gamma+\frac{A}{2} \pm i \frac{1}{2} \sqrt{\Delta \omega^{2}-B^{2}}-i \bar{\omega}
$$

For this case, the intraensemble synchronization takes place simultaneously in the two ensembles since the curves $\gamma_{c+}$ and $\gamma_{c-}$ coincide when $\operatorname{Re}\left(\lambda_{ \pm}\right)$becomes positive. Although there occur two Hopf bifurcations, they happen to be one and the same and hence one will not be able to explain the synchronization region S2. A similar problem occurs also with the D1 synchronization regime for $\Delta \omega^{2}<B^{2}$. Therefore we must conclude that the introduction of coupling/noise asymmetries are crucial to account for certain synchronization phenomena and can never be replaced by the introduction of large difference between the mean frequencies of the two ensembles.

It is therefore essential to take account of possible asymmetry while attempting to model natural systems. Certain phenomena, like those discussed here, are attributable to asymmetries in the interactions.

In this paper, we have investigated the role played by coupling, noise, and phase asymmetries in two coupled phase-oscillator ensembles. We have identified a global clustering phenomenon that may be characteristic of either the coupling or the noise asymmetry when the other is absent. Phase asymmetry reduces the likelihood of global clustering and also introduces routes that are characteristic of itself. Thus phase asymmetry controls the routes to interensemble synchronization. The phenomenon of interensemble clustering that is characteristic of coupling asymmetry is found to occur even for symmetrically coupled systems if noise asymmetry is present. Thus noise asymmetry is found to complement the effect of coupling asymmetry when the latter is absent.

We therefore conclude that, in modeling real systems where synchronization arises, explicit consideration should be given to the effect of possible asymmetries in coupling, noise, and phase.

\section{ACKNOWLEDGMENTS}

The study was supported by the EC FP6 NEST-Pathfinder project BRACCIA and in part by the Slovenian Research Agency, the Engineering and Physical Sciences Research Council (UK), and the DST Ramanna program (Government of India).
[1] J. Buck and E. Buck, Science 159, 1319 (1968).

[2] C. S. Peskin, Mathematical Aspects of Heart Physiology (Courant Institute of Mathematical Sciences, New York, 1975).

[3] I. Z. Kiss, Y. M. Zhai, and J. L. Hudson, Phys. Rev. Lett. 88, 238301 (2002).

[4] I. Z. Kiss, Y. M. Zhai, and J. L. Hudson, Science 296, 1676 (2002).

[5] D. Hansel and H. Sompolinsky, Phys. Rev. Lett. 68, 718 (1992).

[6] P. C. Bressloff, Phys. Rev. E 60, 2160 (1999).
[7] D. Golomb, D. Hansel, and G. Mato, in Handbook of Biological Physics, edited by F. Moss and S. Gielen (Elsevier, Amsterdam, 2001), Vol. 4, pp. 887-968.

[8] T. J. Walker, Science 166, 891 (1969).

[9] A. T. Winfree, J. Theor. Biol. 16, 15 (1967).

[10] Y. Kuramoto, Chemical Oscillations, Waves, and Turbulence (Springer-Verlag, Berlin, 1984).

[11] S. H. Strogatz, Physica D 143, 1 (2000).

[12] S. H. Strogatz, Nature (London) 410, 268 (2001).

[13] A. Pikovsky, M. Rosenblum, and J. Kurths, 
Synchronization-A Universal Concept in Nonlinear Sciences (Cambridge University Press, Cambridge, 2001).

[14] Jane H. Sheeba, V. K. Chandrasekar, A. Stefanovska, and P. V. E. McClintock, Phys. Rev. E 78, 025201(R) (2008).

[15] A. T. Winfree, The Geometry of Biological Time (Springer, Berlin, 1980).

[16] A. Pikovsky, M. Rosenblum, and J. Kurths, Europhys. Lett. 34, 165 (1996).

[17] N. F. Rulkov, Phys. Rev. Lett. 86, 183 (2001).

[18] H. Haken, Advanced Synergetics: Instability Hierarchies of Self-Organizing Systems (Springer, Berlin, 1993).

[19] Z. Néda, E. Ravasz, Y. Brechet, T. Vicsek, and A. L. Barabasi, Nature (London) 403, 849 (2000).

[20] D. Topaj, W.-H. Kye, and A. Pikovsky, Phys. Rev. Lett. 87, 074101 (2001).

[21] B. Blasius, E. Montbrio, and J. Kurths, Phys. Rev. E 67, 035204(R) (2003).

[22] E. Montbrio and B. Blasius, Chaos 13, 291 (2003).

[23] B. Blasius, Phys. Rev. E 72, 066216 (2005).

[24] B. R. Trees, V. Saranathan, and D. Stroud, Phys. Rev. E 71, 016215 (2005).

[25] F. Rogister and R. Roy, Phys. Rev. Lett. 98, 104101 (2007).

[26] C. Schafer, M. G. Rosenblum, J. Kurths, and H. H. Abel, Nature (London) 392, 239 (1998).

[27] M. B. Lotrič and A. Stefanovska, Physica A 283, 451 (2000).

[28] W. Singer, Nature (London) 397, 391 (1999).

[29] W. Singer, Neuron 24, 49 (1999).

[30] P. Fries, Trends Cogn. Sci. 9, 474 (2005).

[31] Y. Yamaguchi, N. Sato, H. Wagatsuma, Z. Wu, C. Molter, and Y. Aota, Curr. Opin. Neurobiol. 17, 197 (2007).

[32] Jane H. Sheeba, A. Stefanovska, and P. V. E. McClintock, Biophys. J. 95, 2722 (2008).

[33] J. A. Goldberg, T. Boraud, S. Maraton, S. N. Haber, E. Vaadia, and H. Bergman, J. Neurosci. 22, 4639 (2002).

[34] L. Timmermann, J. Gross, M. Dirks, J. Volkmann, H. Freund, and A. Schnitzler, Brain 126, 199 (2003).

[35] B. Percha, R. Dzakpasu, M. Zochowski, and J. Parent, Phys.
Rev. E 72, 031909 (2005).

[36] M. Zucconi, M. Manconi, D. Bizzozero, F. Rundo, C. J. Stam, L. Ferini-Strambi, and R. Ferri, Neurol. Sci. 26, s199 (2005).

[37] A. Stefanovska and M. Bračič, Contemp. Phys. 40, 31 (1999).

[38] M. Paluš and A. Stefanovska, Phys. Rev. E 67, 055201(R) (2003).

[39] B. Musizza, A. Stefanovska, P. V. E. McClintock, M. Paluš, J. Petrovčič, S. Ribarič, and F. F. Bajrović, J. Physiol. (London) 580, 315 (2007).

[40] H. Daido and K. Nakanishi, Phys. Rev. Lett. 93, 104101 (2004).

[41] H. Daido and K. Nakanishi, Phys. Rev. Lett. 96, 054101 (2006).

[42] H. Daido and K. Nakanishi, Phys. Rev. E 75, 056206 (2007).

[43] H. Fukuda, N. Nakamichi, M. Hisatsune, H. Murase, and T. Mizuno, Phys. Rev. Lett. 99, 098102 (2007).

[44] A. Sherman and J. Rinzel, Proc. Natl. Acad. Sci. U.S.A. 89, 2471 (1992).

[45] P. R. Roelfsema, A. K. Engel, P. Konig, and W. Singer, Nature (London) 385, 157 (1997).

[46] K. Okuda and Y. Kuramoto, Prog. Theor. Phys. 86, 1159 (1991).

[47] E. Montbrio, J. Kurths, and B. Blasius, Phys. Rev. E 70, 056125 (2004)

[48] P. Tass, Phys. Rev. E 56, 2043 (1997).

[49] Y. Chembo Kouomou and P. Woafo, Phys. Rev. E 67, 046205 (2003).

[50] D. He, P. Shi, and L. Stone, Phys. Rev. E 67, 027201 (2003).

[51] D. S. Goldobin, and A. Pikovsky, Phys. Rev. E 71, 045201(R) (2005).

[52] H. Nakao, K. Arai, and Y. Kawamura, Phys. Rev. Lett. 98, 184101 (2007).

[53] Y. Kawamura, H. Nakao, and Y. Kuramoto, Phys. Rev. E 75, 036209 (2007)

[54] Y. Kawamura, H. Nakao, K. Arai, H. Kori, and Y. Kuramoto Phys, Phys. Rev. Lett. 101, 024101 (2008). 